\title{
Validación de constructo de la escala ESTE-R para medición de la soledad en la vejez en Bucaramanga, Colombia*
}

\author{
Construct Validation of the ESTE-R Scale for Measuring \\ Loneliness in Old Age in Bucaramanga, Colombia
}

\author{
Ara Mercedes Cerquera Córdoba** \\ María Lucía Cala Rueda \\ Mayra Juliana Galvis Aparicio \\ Universidad Pontificia Bolivariana, \\ Bucaramanga \\ Recibido: 2 de diciembre de 2011 \\ Revisado: 4 de marzo de 2012 \\ Aceptado: 30 de mayo de 2012
}

\section{Resumen}

Existen múltiples instrumentos que permiten medir propiedades que aunque no son observables, son de vital importancia para comprender la conducta humana y el trabajo con esta. La soledad es una condición que afecta a todos los grupos de edad; sin embargo, en la vejez hay condiciones que pueden favorecer su aparición, y sus consecuencias pueden ser más temidas y fuertes que en otras etapas de la vida, constituyéndose así en un fenómeno de interés para ser estudiado e intervenido. La escala ESTE-R es un instrumento útil en la medición de la soledad, pero este debe ser validado en el contexto en que sea aplicado, para obtener así resultados confiables. Por esta razón se realizó un estudio instrumental, con una muestra de 383 personas mayores residentes en Bucaramanga, Colombia. Se hizo la medición del alfa de Cronbach y el análisis factorial, obteniéndose los siguientes resultados: elevada fiabilidad $(0,925)$ para todas las variables de la escala y una solución de 4 factores que explican el $58,6 \%$ de la varianza de los datos. Se concluye que la estructura original de cuatro factores es adecuada para el contexto bumangués y que la propuesta de treinta ítems aquí presentada permite medir el fenómeno de la soledad.

Palabras clave: soledad, escala ESTER-R, vejez, validez, confiabilidad.

* $\quad$ Articulo de investigación

** Correspondencia: Ara Mercedes Cerquera Córdoba, Universidad Pontificia Bolivariana, Bucaramanga, Santander, Colombia. Dirección postal: Calle 94 No. 47 89, Santa Bárbara 1 casa 9, Bucaramanga, Colombia. Correos electrónicos: aramercedes@yahoo.com, mayra.galvis@upb.edu.co 


\section{Abstract}

There are many instruments that measure properties that are not observable, but are very important for understanding human behavior and work with it. Loneliness is a condition that affects all age groups, however, in old age there are conditions that may favor its occurrence and its consequences may be more feared and stronger than in other stages of life, becoming in a phenomenon of interest to be studied and intervened. ESTE-R scale is a useful tool for measuring loneliness, but must be validated to the context it is applied to obtain reliable results. For this reason was realized an Instrumental study, with a sample of 383 elder people residents in Bucaramanga, Colombia. Measurement was performed by Cronbach's alpha and factor analysis, obtaining the following results: High reliability $(0,925)$ for all scale variables and a solution of 4 factors that explain $58,6 \%$ of the data variance. Was concluded that the original structure of 4 factors is appropriate for Bucaramanga's context and the proposal of 30 items presented here lets measure loneliness phenomenon.

Keywords: loneliness, ESTE-R scale, old age, validity, reliability.

\section{Introducción}

La soledad se ha estudiado desde diferentes enfoques, y a partir de estos han surgido diversas definiciones del término, haciendo que este se convierta en un concepto complejo que agrupa elementos de orden psicológico y social (Sánchez, 2009; Rubio, 2004).

De este constructo, Rubio y Aleixandre (2001) consideran importante realizar una distinción entre estar solo y sentirse solo, asociándose lo primero al aislamiento social, la carencia de redes sociales, la marginación y el desarraigo; y lo segundo, a la verdadera soledad, pues es el sentimiento que genera nostalgia, tristeza, añoranza, etc., aunque se esté materialmente acompañado de personas.

Teniendo en cuenta lo anterior, una de las definiciones que mejor ilustra el término "soledad" es la que refieren H. Rubio, P. M. Rubio y R. L. Rubio (2009), que la asumen como una condición de malestar emocional, producto de un sentimiento de incomprensión y rechazo de parte de los otros, o de la falta de compañía para la realización de actividades o el logro de intimidad emocional. Así, la soledad es una sensación de carencia del afecto deseado, que produce sufrimiento, desolación, insatisfacción y angustia, y que como indica Rodríguez (2009), nunca es una situación buscada.

\section{Soledad en la vejez}

La soledad es una situación que puede afectar a todos los grupos de edad, y seguramente todos los seres humanos la han experimentado en algún momento de la vida; sin embargo, en la vejez ciertas condiciones pueden favorecer su fácil aparición, y sus consecuencias son más temidas e incluso más fuertes que en otras edades (Carvajal y Caro, 2009; Rubio y Aleixandre, 2001).

Dentro de las condiciones que favorecen la soledad se encuentran, por ejemplo, la disminución de los niveles de socialización, pues la red social de los mayores puede verse disminuida a causa de la muerte o enfermedad de quienes la componen; las dificultades económicas; la aparición de enfermedades; la menor posibilidad de establecer nuevos vínculos, o la mínima motivación para mantener los ya existentes (Durán et ál., 2008; Rubio, 2009; Sánchez, 2009; Winningham y Pike, 2007).

Dentro de los factores que cooperan en la disminución de la socialización se encuentra la jubilación, pues implica un drástico cambio en la distribución del tiempo y de las relaciones sociales de la persona que, probablemente, giraban en torno al ámbito laboral. Además, esta desvinculación trae como consecuencia cambios de roles, de estatus, reducción de los ingresos, entre otros, 
lo cual puede llevar a la aparición de sentimientos de inutilidad, tristeza, pasividad y soledad; y aún más, si sumado a esto se presentan situaciones familiares adversas o complicaciones de salud (Iglesias et ál., 2001; Torre, 2000).

Igualmente, otro factor de importancia para el desarrollo de sentimientos de soledad es la viudez, ya que implica el final de una relación de intimidad altamente significativa. Adaptarse a ella requiere de fortaleza personal y apoyo social, que incluso puede resultar insuficiente para asimilarla. Además, en el caso de que la persona mayor viviera solo con su pareja, tras su fallecimiento existe la necesidad de decidir si de ahí en adelante continuará viviendo solo, residirá con algún familiar o ingresará a una institución para adultos mayores (Sánchez, 2009; Iglesias et ál., 2001).

La institucionalización, por su parte, a pesar de ser una opción válida para las personas mayores que requieren cuidados especiales o no pueden permanecer con sus familias, puede constituirse en un factor predisponente a sentimientos de soledad, pues como indican Mella et ál. (2004), esta condición favorece la pérdida de relaciones sociales, disminuyendo así el apoyo social percibido como necesario para hacer frente a los diversos cambios que implica el envejecer.

Lo anterior atrae la atención de otro aspecto de gran relevancia para la aparición de sentimientos de soledad en la vejez, a saber: la salud, debido a que un deterioro de esta predispone a dichos sentimientos negativos, los cuales, a su vez, pueden conducir a hábitos poco sanos y a una visión más negativa del propio estado de salud. De igual modo, la soledad trae consigo consecuencias negativas como el deterioro físico y funcional, que puede llevar posteriormente a deterioro cognitivo y a la aparición de problemas como la depresión y la ansiedad, lo que entra en relación con el deterioro de la calidad de vida y con el aumento de la mortalidad (Alpass y Neville, 2003; Luanaigh y Lawlor, 2008; Routasalo y Pitakala, 2003; Rubio, 2004; Sánchez, 2009).

\section{Medición de la soledad en las personas mayores}

Es evidente que la soledad es un fenómeno que puede tener un alto impacto sobre la calidad de vida de las personas mayores, por lo cual resulta importante ahondar en el estudio de esta problemática, generando nuevos conocimientos y aprendizajes que ayuden a crear soluciones de tipo permanente y no temporal. Es de vital importancia contar con herramientas como las escalas de medición, instrumentos que en pro del correcto desarrollo investigativo deben encontrarse validados de manera adecuada y completa. Ejemplo de estos procesos son investigaciones como las realizadas por Zegers, Rojas y Forster (2009), que desarrollaron un estudio sobre la estimación de la confiabilidad y la validez del Índice de Satisfacción Vital (LSI-A) de Neugarten, Havighurst y Tobin; o el estudio hecho sobre propiedades psicométricas y validación de la Escala de Estrategias de Coping Modificada (EEC-M) en una muestra colombiana, efectuado por Londoño et ál. (2006), en el que se identificaron las propiedades psicométricas y la validez estructural de la escala.

Para el ámbito de la soledad se han diseñado escalas de medición utilizadas para su estudio, las cuales surgen debido a que la soledad es considerada como un factor que afecta la calidad de vida de las personas mayores $\mathrm{y}$, adicionalmente, el interés de diversos investigadores en dicho constructo. Algunas de las escalas más utilizadas son las escalas UCLA, SELSA, ESLY y la Escala de Satisfacción Vital de Philadelphia (Borges et ál., 2008; Rubio, 2004).

Tomando como base dichas escalas e incluyendo indicadores subjetivos, más el análisis de redes sociales, la Universidad de Granada (España) diseñó la escala ESTE, asumiendo la soledad como un constructo multidimensional y centrándose en los déficits en el soporte social de los mayores y en el sentimiento que ellos pueden provocar (Rubio, 2004). En 2009, Rubio realizó el proceso de revisión de dicha escala diseñada para medir la soledad en adultos mayores, conformada por 34 ítems tipo Likert, con 5 opciones de respuesta; nunca, 
rara vez, algunas veces, a menudo y siempre. Los ítems de la escala se encontraban agrupados en cuatro factores: soledad familiar, soledad conyugal, soledad social y crisis existencial. A partir de este proceso se encontró una elevada consistencia interna y fiabilidad $(0,915)$, medida con la ayuda alfa de Cronbach.

Procesos de revisión como el anterior llevan a cuestionar la importancia de la validez de la escala, convertida en el aspecto de la medición psicopedagógica que se encuentra vinculado con la comprobación y estudio del significado de las puntuaciones que obtienen los test; en otras palabras, se refiere al grado en que un instrumento realmente mide la variable que pretende evaluar (Anastasi, 1981; Brown, 1976; Hernández, Fernández y Baptista, 1991).

Por tanto, teniendo en cuenta que actualmente la psicología centra su estudio en el examen de las variables que son definidas en y por el test, y que a su vez son comparadas con las externas y observadas con el fin de lograr sustentar la interpretación de las propuestas, Elosua (2003) refiere tres etapas que conforman los estándares de validez: la primera etapa es aquella conocida como operacional, en la que prima la validez de tipo externo y que refiere cuatro tipos de validez: de contenido, predictiva, concurrente y de constructo. Este último tipo se refiere a qué tan exitosamente un instrumento representa y mide un concepto teórico (Grinnell, Williams y Unrau, 2009, citado en Hernández et ál., 2011). A esta validez le concierne en particular el significado del instrumento, esto es, qué está midiendo y cómo opera para medirlo, integrando la evidencia que soporta la interpretación del sentido que poseen las puntuaciones del instrumento.

La segunda etapa tiene que ver con lo teórico. Este aspecto se encuentra enmarcado en la importancia de poseer una visión integradora. Allí el análisis de la validez de constructo se concibe como un concepto unificado que contiene los aspectos relacionados con el contenido y la relación con las demás variables, donde la validez se reconoce como un proceso único de recolección de evidencias a través de múltiples estrategias de investigaciones diferentes relacionadas con el concepto. Finalmente, aparece la tercera fase contextual, en donde el objetivo es dotar a los test con los avales tanto de tipo científico como ético.

Adicional a la validez, un instrumento de medición necesita indicar el grado de estabilidad de los datos a través del tiempo, es decir, debe ser confiable. Esta confiabilidad puede determinarse mediante procedimientos como la estabilidad (ANOVA), el método de formas alternativas o paralelas, el método de mitades partidas y medidas de consistencia interna o coherencia. Este último procedimiento puede realizarse a través de varios coeficientes que miden la confiabilidad, entre ellos el alfa de Cronbach y los de KuderRichardson KR-20 y KR-21. El método de cálculo en ambos casos requiere una sola administración del instrumento de medición, en el cual el resultado es afectado por la heterogeneidad de los individuos evaluados: cuanto más heterogéneo es el grupo, mayor es el coeficiente de fiabilidad (Hernández et ál., 1991).

Los anteriores procesos permiten cuantificar las características humanas propias de la población y, lo más importante, objetivizar los procedimientos de evaluación y su confiabilidad (Malo, 2008). Este último procedimiento se efectúa examinando la dimensionalidad del instrumento, centrándose en realizar el análisis de fiabilidad en lo referente a la consistencia interna y obteniendo la validez convergente y diferencial del instrumento. Es de vital importancia conocer a fondo las características sociodemográficas de la región en la cual se va a validar la herramienta, puesto que estas validaciones de tipo intercultural son un proceso complejo (Balluerka et ál., 2007; Hamblenton, 2005).

Validar instrumentos finalmente termina siendo una de las mejores herramientas, pues permite obtener la información de los principales implicados: los que vivencian directamente la situación. Es por esto que siguiendo los pasos de la validación, se creó una versión de la escala ESTE-R, adaptada al contexto bumangués, con el fin de trabajar el constructo de la soledad en la vejez de la ciudad, y, a partir de los resultados obtenidos con el trabajo realizado con ella, generar nuevos 
procesos investigativos y modelos de intervención encaminados a lograr mejorar la calidad de vida de la persona mayor. Esto configura un constructo complejo, multifactorial, en el cual se busca que la persona se encuentre satisfecha y se sienta productiva en los ámbitos social, físico y psíquico, y se vea impactada positivamente (González, 2006).

\section{Metodología}

Teniendo en cuenta que el fenómeno de la soledad no puede ser observado directamente y que su estudio requiere de indicadores observables, como las respuestas que dan las personas a preguntas conglomeradas dentro de una escala (Carretero-Dios y Pérez, 2005, 2007), el tipo de diseño elegido fue instrumental, el cual contempla acciones de desarrollo de pruebas y aparatos, e incluye también el diseño y la adaptación de instrumentos (Montero y León, 2002).

\section{Muestra}

Fueron seleccionados 383 personas mayores de forma aleatoria. Los criterios de inclusión fueron: personas de ambos géneros, de 65 años de edad en adelante, residentes en la ciudad de Bucaramanga, pertenecientes a grupos de tercera edad de la ciudad, que no presentaran dificultad para comprender los ítems del instrumento, firmaran el consentimiento informado y estuvieran dispuestos a participar en el estudio. Criterios de exclusión: personas con limitación física, sensorial o cognitiva evidente que no les permitiera comprender el instrumento; residentes en centros de bienestar del anciano $u$ otras instituciones para personas mayores de la ciudad. El recurso utilizado en la presente investigación fue el software IBM - SPSS 19 para análisis predictivo.

\section{Resultados}

La escala, después de ser revisada por jueces, quedó conformada por 30 ítems distribuidos de la siguiente manera: soledad familiar (12 ítems), soledad conyugal (5 ítems), soledad social (5 ítems) y crisis existencial (8 ítems) (tabla 1).
Tabla 1.

Distribución de items por componentes: análisis de fiabilidad

\begin{tabular}{lcl}
\hline \multicolumn{1}{c}{ Factor } & Ítem & Puntuación \\
\hline $\begin{array}{l}\text { Soledad } \\
\text { familiar }\end{array}$ & $\begin{array}{c}1,2,8,9,10,11,12, \\
16,17,18,19,20\end{array}$ & $\begin{array}{l}\text { Máxima: } 60 \\
\text { Mínima: } 12\end{array}$ \\
$\begin{array}{l}\text { Soledad } \\
\text { conyugal }\end{array}$ & $3,4,5,6,7$ & $\begin{array}{l}\text { Máxima: } 25 \\
\text { Mínima: } 5\end{array}$ \\
$\begin{array}{l}\text { Soledad } \\
\text { social }\end{array}$ & $14,15,21,22,23$ & $\begin{array}{l}\text { Máxima: } 25 \\
\text { Mínima: } 5\end{array}$ \\
$\begin{array}{l}\text { Crisis } \\
\text { existencial }\end{array}$ & $13,24,25,26,27,28$, & $\begin{array}{l}\text { Máxima: } 40 \\
\text { Mínima: } 8\end{array}$ \\
\hline
\end{tabular}

Fuente: autores.

El análisis de confiabilidad se realizó a través del alfa de Cronbach, que arrojó resultados así: 0,925 para todas las variables de la escala; 0,932 para soledad familiar; 0,887 para soledad conyugal; 0,844 para soledad social, y 0,755 para crisis existencial (tabla 2).

Tabla 2.

Alfa de Cronbach general y por factores

\begin{tabular}{lc}
\hline \multicolumn{1}{c}{ Factor } & Alfa de Cronbach \\
\hline General & 0,925 \\
Soledad familiar & 0,932 \\
Soledad conyugal & 0,887 \\
Soledad social & 0,844 \\
Crisis existencial & 0,755 \\
\hline
\end{tabular}

Fuente: autores.

\section{Validez}

La validez de la escala se valoró a través del análisis factorial, utilizando el método de componentes principales con rotación Varimax, aplicándose primero el índice de Kaiser-Meyer-Olkin, teniendo en cuenta que siendo mayor a 0,7 se podría realizar el análisis factorial; igualmente, se aplicó la prueba de esfericidad de Barlett, obteniéndose 
en esta una significancia de 0,000 y un índice KMO de 0,916 , los cuales indicaron que era posible continuar con el análisis factorial.

Tabla 3.

Varianza total explicada por cuatro factores para la escala ESTE- $R$

\begin{tabular}{|c|c|c|c|c|c|c|}
\hline \multirow{2}{*}{ 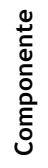 } & \multicolumn{3}{|c|}{ Autovalores iniciales } & \multicolumn{3}{|c|}{$\begin{array}{l}\text { Suma de las saturaciones del } \\
\text { cuadrado de la rotación }\end{array}$} \\
\hline & Total & $\begin{array}{c}\% \text { de la } \\
\text { varian- } \\
\text { za }\end{array}$ & $\begin{array}{l}\% \text { acu- } \\
\text { mulado }\end{array}$ & Total & $\begin{array}{l}\% \text { de la } \\
\text { varianza }\end{array}$ & $\begin{array}{l}\% \text { acumu- } \\
\text { lado }\end{array}$ \\
\hline 1 & 10,325 & 34,417 & 34,417 & 7,209 & 24,029 & 24,029 \\
\hline 2 & 3,264 & 10,879 & 45,297 & 3,967 & 13,224 & 37,253 \\
\hline 3 & 2,322 & 7,739 & 53,035 & 3,731 & 12,438 & 49,691 \\
\hline 4 & 1,686 & 5,622 & 58,657 & 2,690 & 8,966 & 58,657 \\
\hline 5 & 1,264 & 4,213 & 62,869 & & & \\
\hline 6 & 1,097 & 3,656 & 66,525 & & & \\
\hline 7 & 0,998 & 3,327 & 69,852 & & & \\
\hline 8 & 0,830 & 2,767 & 72,619 & & & \\
\hline 9 & 0,767 & 2,557 & 75,176 & & & \\
\hline 10 & 0,686 & 2,286 & 77,463 & & & \\
\hline 11 & 0,635 & 2,117 & 79,580 & & & \\
\hline 12 & 0,614 & 2,045 & 81,625 & & & \\
\hline 13 & 0,562 & 1,872 & 83,497 & & & \\
\hline 14 & 0,520 & 1,732 & 85,229 & & & \\
\hline 15 & 0,459 & 1,532 & 86,761 & & & \\
\hline 16 & 0,426 & 1,419 & 88,179 & & & \\
\hline 17 & 0,410 & 1,367 & 89,546 & & & \\
\hline 18 & 0,392 & 1,308 & 90,854 & & & \\
\hline 19 & 0,335 & 1,117 & 91,971 & & & \\
\hline 20 & 0,324 & 1,081 & 93,052 & & & \\
\hline 21 & 0,293 & 0,976 & 94,029 & & & \\
\hline 22 & 0,282 & 0,940 & 94,969 & & & \\
\hline 23 & 0,263 & 0,878 & 95,847 & & & \\
\hline 24 & 0,244 & 0,814 & 96,661 & & & \\
\hline 25 & 0,229 & 0,762 & 97,423 & & & \\
\hline 26 & 0,197 & 0,657 & 98,080 & & & \\
\hline 27 & 0,181 & 0,604 & 98,684 & & & \\
\hline 28 & 0,152 & 0,507 & 99,191 & & & \\
\hline 29 & 0,136 & 0,452 & 99,643 & & & \\
\hline 30 & 0,107 & 0,357 & 100,000 & & & \\
\hline
\end{tabular}

Fuente: autores.

El análisis factorial de la escala mostró una solución de cuatro elementos que explican el 58,6\% de la varianza de los datos, siendo el componente 1 el que explica en mayor proporción la varianza, con un $34,4 \%$ (tabla 3 ).
Tabla 4.

Solución factorial de la escala

\begin{tabular}{|c|c|c|c|c|}
\hline \multirow{2}{*}{ Variable } & \multicolumn{4}{|c|}{ Componente } \\
\hline & 1 & 2 & 3 & 4 \\
\hline $\mathrm{p} 1 \mathrm{~F}$ & 0,422 & & & \\
\hline $\mathrm{p} 2 \mathrm{~F}$ & 0,566 & & & \\
\hline $\mathrm{p} 8 \mathrm{~F}$ & & & & \\
\hline $\mathrm{p} 9 \mathrm{~F}$ & 0,813 & & & \\
\hline $\mathrm{p} 10 \mathrm{~F}$ & 0,847 & & & \\
\hline $\mathrm{p} 11 \mathrm{~F}$ & 0,832 & & & \\
\hline $\mathrm{p} 12 \mathrm{~F}$ & 0,797 & & & \\
\hline $\mathrm{p} 16 \mathrm{~F}$ & 0,831 & & & \\
\hline p17F & 0,746 & & & \\
\hline $\mathrm{p} 18 \mathrm{~F}$ & 0,462 & & 0,471 & \\
\hline $\mathrm{p} 19 \mathrm{~F}$ & 0,758 & & & \\
\hline $\mathrm{p} 20 \mathrm{~F}$ & 0,784 & & & \\
\hline p3C & & 0,698 & & \\
\hline $\mathrm{p} 4 \mathrm{C}$ & & 0,862 & & \\
\hline $\mathrm{p} 5 \mathrm{C}$ & & 0,872 & & \\
\hline p6C & & 0,650 & & \\
\hline p7C & & 0,875 & & \\
\hline p14S & & & & 0,728 \\
\hline p15S & & & & 0,762 \\
\hline p21S & 0,581 & & & 0,472 \\
\hline p22S & & & & 0,708 \\
\hline p23S & 0,540 & & & 0,611 \\
\hline p13CE & & & & \\
\hline p24CE & & & 0,585 & \\
\hline p25CE & & & 0,604 & \\
\hline p26CE & & & 0,591 & \\
\hline p27CE & & & 0,752 & \\
\hline p28CE & & & 0,771 & \\
\hline p29CE & & & 0,722 & \\
\hline p30CE & & & 0,418 & \\
\hline
\end{tabular}

Fuente: autores. 
La tabla 4 muestra la solución factorial de la escala: se observa que el primer factor está constituido por once de las doce variables de soledad familiar, ya que el ítem 8 no discrimina en ningún factor; sin embargo, el ítem 18 presenta una doble saturación, discriminando además para el factor 3. Igualmente, las variables 21 y 23 , que corresponden a soledad social, discriminan para el primer factor.

El factor 2 agrupa las cinco variables referidas a soledad conyugal. Por otra parte, el factor 3 agrupa las siete variables de crisis existencial y una variable de soledad familiar que discrimina además para el primer factor, pues presenta doble saturación. Finalmente, el factor 4 está conformado por las cinco variables de soledad social, aunque dos de ellas (p21 y p23) discriminan también para el factor 1. La variable 13, que corresponde a crisis existencial, no discrimina para ningún factor.

Los ítems 8 y 13 no discriminan para ningún factor, presentan saturaciones por debajo de 0,4 (3,72 y 0,84 , respectivamente) y los ítems 18,21 y 23 discriminan para más de un factor.

\section{Discusión}

Se realizó la validación de la escala ESTE-R en el contexto bumangués, la cual, después de haber sido revisada por jueces, quedó conformada a nivel general por 30 ítems, diferenciándose de la escala original compuesta por 34 , distribuidos de la siguiente forma: soledad familiar (12 ítems), soledad conyugal (5 ítems), soledad social (5 ítems) y crisis existencial (8 ítems).

El alfa de Cronbach obtenido para toda la escala fue de 0,925 , encontrándose una alta confiabilidad coincidente con la resultante en las investigaciones realizadas en España por Rubio (2009) y en Bello, Antioquia, por Cardona, Villamil, Henao y Quintero (2010), con resultados de 0,915 y 0,90, respectivamente.

Los valores de alfa de Cronbach obtenidos en cada subescala se encuentran entre 0,932 y 0,755 , siendo el factor soledad familiar el que mayor confia- bilidad presenta y crisis existencial el de menor confiabilidad.

Por otro lado, el factor soledad familiar obtuvo un coeficiente de alfa de Cronbach de 0,932, mientras que en el estudio de Cardona et ál. (2010) se encontró un valor de 0,82. La diferencia existente entre los dos coeficientes es amplia, lo que permite evidenciar que una mayor cantidad de ítems posibilita una mejor medición del componente de soledad familiar, puesto que la subescala de la presente investigación contó con doce ítems, en tanto la realizada en Antioquia solo presentaba cuatro.

El factor soledad conyugal estuvo constituido en los instrumentos de ambas investigaciones por cinco ítems y obtuvo un coeficiente de alfa de Cronbach de 0,887 en Bucaramanga y de 0,91 en Antioquia, evidenciando que la cantidad de ítems de dicha subescala permiten medir apropiadamente este tipo de soledad. La diferencia en los coeficientes de ambas investigaciones podría estar relacionada con la cantidad de personas que conformaron la muestra (Bucaramanga: 383 adultos; y Bello, Antioquia: 180 adultos).

Por otra parte, la soledad social obtuvo coeficiente de alfa de Cronbach de 0,844 y estuvo compuesta por cinco ítems; por su parte, en el estudio de Cardozo et ál. (2010) se obtuvo un valor de 0,85 y contó con ocho ítems, lo cual indica que la cantidad de ítems en este caso no afecta la confiabilidad en la medición de este componente.

El coeficiente de alfa de Cronbach que obtuvo el factor crisis existencial, compuesto por ocho ítems, fue de 0,755 , contrario a lo encontrado en Bello, Antioquia, donde se obtuvo un valor de 0,89 y estaba conformado por trece ítems. Esto permite evidenciar nuevamente que al estar constituida la subescala por mayor cantidad de ítems, la confiabilidad en la medición de este componente es mayor.

Al igual que la escala original (española) y la escala validada en Bello, Antioquia, el análisis factorial realizado a la escala en el contexto bumangués muestra una solución de cuatro factores, siendo el primero de ellos el que explica en mayor 
proporción la varianza de los datos. A través de este análisis se encontró, además, que los ítems 8 y 13 , correspondientes a soledad familiar y crisis existencial, respectivamente, no discriminan para ninguno de los factores, mientras que el ítem 18 correspondiente a soledad familiar, y los ítems 21 y 23 pertenecientes a soledad social presentan doble saturación, haciéndose necesaria su revisión y eliminación según corresponda.

En conclusión, la estructura factorial de cuatro componentes indicada por Rubio (2009) es adecuada para la medición de la soledad en el contexto bumangués. Igualmente, salvo los ítems mencionados anteriormente que presentan saturaciones bajas o dobles, la propuesta de treinta ítems presentada en esta investigación abstrae información confiable sobre el fenómeno que pretende medir.

Finalmente, los resultados de la presente investigación muestran que la escala ESTE-R es un instrumento útil para el estudio de la soledad en la tercera edad en Bucaramanga, mostrando una alta confiabilidad y validez; a partir de lo cual pueden realizarse nuevas investigaciones sobre dicho fenómeno que posibiliten generar nuevos conocimientos y estrategias de intervención para el mejoramiento de la calidad de vida de los adultos mayores de la región.

\section{Referencias}

Alpass, F. M. \& Neville, S. (2003). Loneliness, health and depression in older males. Aging and Mental Health, 7(3), 212-216.

Anastasi, A. (1981). Capacitación, avance de las pruebas y capacidades desarrolladas. México: Trillas.

Balluerka, N., Gorostiaga, A., Arbiol, I. y Haranburu, M. (2007). La adaptación de instrumentos de medida de unas culturas a otras: una perspectiva práctica. Psicothema 19(1), 124-133.
Borges, A., Prieto, P., Ricchetti, G., Hernández, C. y Rodríguez, E. (2008). Validación cruzada de la factorización del Test UCLA de soledad. Psicothema, 20(4), 924-927.

Brown, W. (1976). Prácticas actuales de la evaluación psicológica. México: Manual Moderno.

Cardozo, J., Villamil, M., Henao, E. y Quintero, A. (2010). Validación de la escala ESTE para medir la soledad de la población adulta. Investigación y Educación en Enfermería, 28(3).

Carretero-Dios, H. y Pérez, C. (2005). Normas para el desarrollo y revisión de estudios instrumentales. International journal of Clinical and Health Psychology, 5(3), 521-551.

Carretero-Dios, H. y Pérez, C. (2007). Normas para el desarrollo y revisión de estudios instrumentales: consideraciones sobre la selección de test en la investigación psicológica. International Journal of Clinical and Health Psychology, 7(3), 863-882.

Carvajal, G. y Caro, C. (2009). Soledad en la adolescencia: análisis del concepto. Aquichan, 9(3), 281-296.

Durán, D., Valderrama, L., Uribe, A. y Uribe, J. (2008). Integración social y habilidades funcionales en adultos mayores. Universitas Psychologica, 7(1), 263-270.

Elosua, P. (2003). Sobre la validez de los test. Psichotema, 15(2), 315-321.

González, A. (2006). Calidad de vida en el adulto mayor. México: Instituto de Geriatría.

Hambleton, R. K. (2005). Issues, designs and technical guidelines for adapting tests into multiple languages and cultures. En R. K. Hambleton, P. F. Merendad \& C. D. Spielberger (Eds.). Adapting psychological and educational tests for cross-cultural assessment. New Jersey: Lawrence Erlbaum. 
Hernández, R., Fernández, C. y Baptista, P. (1991). Metodología de la Investigación. México: McGraw-Hill.

Iglesias, J., López, J., Díaz, M. P., Alemán, C., Requena, T. y Castón, P. (2001). La soledad en las personas mayores: influencias personales, familiares y sociales. Análisis cualitativo. Madrid: Instituto de Migraciones y Servicios Sociales (Imserso).

Londoño, N., Henao, G., Puerta, I., Posada, S., Arango, D. y Aguirre, D. (2006). Propiedades psicométricas y validación de la escala de estrategias de Coping modificada (EEC-M) en una muestra colombiana. Universitas Psychologica, 5(2), 327-349.

Luanaigh, C. \& Lawlor, B. (2008). Loneliness and the health of older people. International Journal of Geriatric Psychiatry, 23, 1213-1221.

Malo, D. (2008). La medición en psicología como herramienta y como reflexión ética en el ejercicio del psicólogo. Psicogente, 11, 46-51.

Mella, R., González, L., D’Appolonio, J., Maldonado, I., Fuenzalida, A. y Díaz, A. (2004). Factores asociados al bienestar subjetivo en el adulto mayor. Psykhe, 5(1), 79-89.

Montero, I. y León, O. (2002). Clasificación de las metodologías de investigación en psicología. International Journal of Clinical and Health Psychology, 2(3), 503-508.

Rodríguez, M. (2009). La soledad en el anciano. Gerokomos, 20(4), 159-166.

Routasalo, P. \& Pitakala, K. (2003). Loneliness among older people. Reviews in Clinical Gerontology, 13(4), 303-311.
Rubio, R. (2004). La soledad en personas mayores españolas. Madrid: Portal Mayores. Recuperado de http://www.imsersomayores. csic.es/documentos/documentos/rubio-soledad-01.pdf

Rubio, R. y Aleixandre, M. (2001). Un estudio sobre la soledad en las personas mayores: entre el estar solo y el sentirse solo. Revista Multidisciplinar de Gerontología, 11(1), 23-28.

Rubio-Herrera, R., Pinel, M. y Rubio-Rubio, L. (2009). La soledad en los mayores: una alternativa de medición a través de la escala ESTE. Granada: Universidad de Granada.

Sánchez, M. (2009). Determinantes sociales de la soledad en personas mayores españolas: Dar y recibir apoyo en el proceso de envejecer (tesis doctoral). España: Universidad de Salamanca de Salamanca.

Torre, J. (2000). Gerobus: una alternativa para combatir la soledad de los mayores Gerokomos, 11(2), 86-92.

Winningham, R. G. \& Pike, N. L. (2007). A cognitive intervention to enhance institutionalized older adults' social support networks and decrease loneliness. Aging and Mental Health, 11(6), 716-721.

Zegers, B., Rojas, B. y Forster, C. (2009). Validez y confiabilidad del Índice de Satisfacción Vital (LSI-A) de Neugarten, Havighurst y Tobin en una muestra de adultos y adultos mayores en Chile. Terapia Psicológica, 27(1), 15-26. 